\title{
Mathematical Model for the Influence of wind load on mooring system
}

\author{
Yikai Wang \\ School of North China Electric Power University , Baoding 071000 , China; \\ 742657004@qq.com
}

Keywords: Static equilibrium equation, Torque balance equation, Iterative Approximation Methods

\begin{abstract}
Mooring system consists of pipes, steel drum, heavy ball, welding chain and a special anti-drag anchor. Due to its ability to tie up the buoy system, the buoy system can work properly under the influence of wind loads. When the mooring system is working normally, the angel of the sea floor and the tangential direction of the connection of anchor chain and anchor cannot exceed 16 degrees. And the inclination angle of steel drum cannot exceed 5 degrees. To solve the parameters of the mooring system, each part in the system needs to be analyzed individually. Suppose the angel of the sea floor and the tangential direction of the connection of anchor chain and anchor is exactly $0^{\circ}$, in this case the draught height of the buoy can be calculated. Now the tension of the steel pipe for the buoy can be solved by static equilibrium equation. Through static equilibrium equation and torque balance equation, the tension of steel pipes and steel drum can also be analyzed. After the tension and inclination of every section are analyzed, the suspension length of the anchor chain can be gained by using catenary equation. Assume it as a new volume, then repeat the above process step by step. Finally, through Iterative Approximation, the true value of the height of draught can be calculated. Using this value, the inclination angle of steel pipes and steel drum can be calculated, so that we can analyze whether the mooring system can work normally with certain wind speed.
\end{abstract}

\section{Introduction}

Transport nodes of near shallow observation network consist of the buoy system $^{[1]}$, mooring system and underwater acoustic communication system. Among them, mooring system consists of pipes, steel drum, heavy ball, welding chain and a special anti-drag anchor. When the mooring system is working normally, the angel of the sea floor and the tangential direction of the connection of anchor chain and anchor cannot exceed 16 degrees. Otherwise, the anchor will be dragged along, causing the loss of node shift. At the same time, if the inclination angle of steel drum exceeds 5 degrees, it will make the underwater acoustic communication system within poor working results. Due to large offshore wind speed, wind load often has an obviously impact on the mooring system. Therefore, in order to judge whether the mooring system can work normally with certain wind speed, we need to judge whether the angel of the sea floor and the tangential direction of the connection of anchor chain and anchor exceeds 16 degrees and whether the inclination angle of steel drum exceeds 5 degrees.

Mooring system consists of pipes, steel drum, heavy ball, welding chain and a special anti-drag anchor. Due to the geometry of every part is various and the forces are not identical, the force of mooring system cannot be analyzed integrally. So the force of each part in the system needs to be analyzed individually.

When the mooring system is working normally, the angel of the sea floor and the tangential direction of the connection of anchor chain and anchor cannot exceed 16 degrees. Suppose the angel is exactly $0^{\circ}$, in other words, all chains are pending. Since the study only researches on the effect of wind loads, so the influence of the current loads is neglected. As for the buoy, due to the joint action of the mooring system and the wind load, finally, it will be in a state of static equilibrium. Now establish its Static equilibrium equations in horizontal and vertical directions.

According to Newton's second law, the Static equilibrium equations in horizontal direction is that

$$
T_{1 x}=T_{1} \cos \beta=F_{W}
$$


where $T_{1}$ means the the tension of the steel pipe for the buoy, $F_{w}$ means wind load, its formula is that

$$
F_{W}=0.625 S v^{2}
$$

where $v$ means the wind speed, $S$ means the projected area of the buoy in the wind normal plane. The Static equilibrium equations in vertical direction is that

$$
T_{1 y}=T_{1} \sin \beta=F-G
$$

where $G$ means the gravity of the buoy, $F$ means the buoyancy of the sea, its formula is that

$$
F=\rho g V_{o}=\rho g S_{d} h_{1}
$$

where $\rho$ means the density of the sea water, $g$ means the acceleration of gravity, $S_{d}$ means the bottom area of the buoy, $h_{1}$ means the draught height of the buoy.

According to the assumption that the angel of the sea floor and the tangential direction of the connection of anchor chain and anchor is exactly $0^{\circ}$, taking into account the role of anchor, its force for other parts of the mooring system is totally in the left horizontal direction. So in the vertical direction, the forces of other parts of the mooring system are gravity and buoyancy. And they satisfy the Equilibrium condition that

$$
\sum F=\sum G
$$

When the size and weight of the buoy, pipes, drums, heavy ball and chain are known, the draught height of the buoy can be calculated by the above formulas.

However, corresponding to different wind speed, the situation of the anchor chain is different. So the value of the draught height of the buoy above is not accurate. It is needed as a basis for the next iteration. According to this value, the gravity, buoyancy and wind power of the buoy can be gained. Through static equilibrium equation, the tension $T_{1}$ of the first steel pipe can be solved. After the forces of the steel pipes are analyzed, it can be found that all sections have the same forces of nature through Recursive Analysis. As for the pipe of section $i$, its forces contain gravity $G_{0}$ and two adjacent pipes for its tensions $T_{i}$ and $T_{i+1}$. The three forces above meet the static equilibrium equations $^{[2]}$ that

$$
\begin{aligned}
T_{i} \cos \beta_{i}=T_{i+1} \cos \beta_{i+1} \\
T_{i} \sin \beta_{i}=T_{i+1} \sin \beta_{i+1}+G_{0}
\end{aligned}
$$

where $\beta_{i}$ means the horizontal angel of the tension $T_{i}, \beta_{i+1}$ means the horizontal angel of the tension $T_{i+1}$. Through Recursive Analysis, all the tensions can be calculated.

However, the horizontal angel of the tension does not equal to its geometry angle $\theta$, from the magnitude point of view, the former is slightly larger than the latter. As for the pipe of section $i$, taking torque at the pipe bottom end point, it satisfies the Torque balance equation that

$$
M_{G}=M_{T}
$$

Substitute variable values, then it will turn into that

$$
\frac{1}{2} G_{0} l_{i} \cos \theta_{i}=T_{i} l_{i} \sin \left(\beta_{i}-\theta_{i}\right)
$$

When the horizontal angel of the tension $\beta$ is known, every geometry angle of steel pipes can be calculated corresponding to different wind speeds.

Because a heavy ball is hanging below the steel drum, the force of the drum cannot be analyzed like before. The tension from the heavy ball and the own gravity of the steel drum have different force points, so they generate two different force torques. The steel drum meets the following Static equilibrium equation and Torque balance equation

$$
\begin{gathered}
T_{j} \cos \beta_{j}=T_{0} \cos \beta_{0} \\
T_{j} \sin \beta_{j}+F=T_{0} \sin \beta_{0}+G_{j}+G_{b 1}
\end{gathered}
$$




$$
\frac{1}{2}\left(G_{j}-F\right) l_{j} \sin \theta_{j}+G_{b 1} l_{j} \sin \theta_{j}=T_{0} l_{j} \cos \left(\beta_{0}-\theta_{j}\right)
$$

Where $T_{j}$ means the tension from the last section of the pipes, $T_{0}$ means the tension from anchor to the steel drum, $G_{b 1}$ means the gravity of the heavy ball, $G_{j}$ means the gravity of the steel drum, $\beta_{j}$ means the horizontal angel of the tension of drum, $\beta_{0}$ means the horizontal angel of the tension of anchor, $\theta_{j}$ means the horizontal geometry angle of the drum. By using these values, the inclination angle of steel drum $\theta_{j}$ can be calculated.

When the draught height of the buoy, the inclination angle of steel drum, and every section of the steel pipes are calculated, the ordinate of the top of the anchor chain $y$ can be calculated. Substitute it into the formula derived from the catenary equation ${ }^{[3]}$

$$
s=\sqrt{y^{2}+\frac{2 y F}{w}}
$$

If $s \leq s_{0}$ ( $\mathrm{s}_{0}$ means the length of the anchor chain), it means that part of the anchor chain is dragging on the ground. Using the value of $s$, the length of the suspending part of chain can be calculated. Repeat the procedure above, a new value of the length of the suspending part of chain can be calculated. Iterate the value step by step, the draught height of the buoy will tend to a stable value. This value can be viewed as the accurate value. When the accurate value corresponding to a certain wind speed is calculated, the angle with the vertical direction of the drums can be calculated through Static equilibrium equation and Torque balance equation. In this case, we can judge whether the mooring system can work normally.

\section{Summary}

When judging whether the mooring system is working normally, we can establish Static equilibrium equation and Torque balance equation to analyze the force. When the pending length of the anchor chain is calculated, viewing is as a known condition, then repeating the process above again and again. Finally, through iterating the value, the accurate value of the draught height of the buoy can be calculated. Then the angle with the vertical direction of the drums can be calculated. Finally, by comparing the calculated value with the limit value, we can judge whether the mooring system is working normally.

\section{References}

[1] Surface Buoy Systems. http://www.mooringsystems.com/surface.htm

[2]Xiaofang Hu, Deyong Ding. The Influence of the ship capacity at anchor chain diameter [J]. Research of China Ship,2014,04:109-112+119.

[3]Fuzhong Bai. Multi-anchor mooring ship stability calculation [J]. Learned journal of He Hai University,1993,02:21-27. 